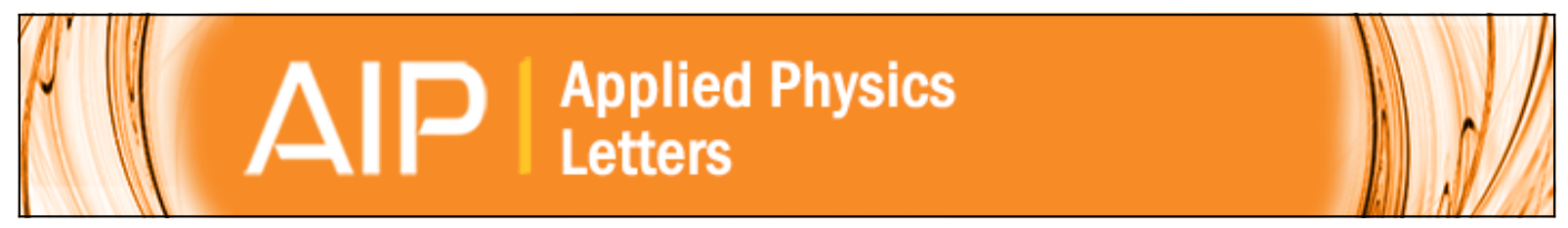

\title{
Atomic-scale depth selectivity of soft x-ray resonant Kerr effect
}

Ki-Suk Lee, Sang-Koog Kim, and J. B. Kortright

Citation: Applied Physics Letters 83, 3764 (2003); doi: 10.1063/1.1622123

View online: http://dx.doi.org/10.1063/1.1622123

View Table of Contents: http://scitation.aip.org/content/aip/journal/apl/83/18?ver=pdfcov

Published by the AIP Publishing

\section{Articles you may be interested in}

Soft x-ray resonant magneto-optical Kerr effect as a depth-sensitive probe of magnetic heterogeneity: A simulation approach

J. Appl. Phys. 97, 083519 (2005); 10.1063/1.1861969

Soft x-ray resonant Kerr rotation measurement and simulation of element-resolved and interface-sensitive magnetization reversals in a $\mathrm{Ni} \mathrm{Fe} / \mathrm{Fe} \mathrm{Mn} / \mathrm{Co}$ trilayer structure

Appl. Phys. Lett. 86, 102502 (2005); 10.1063/1.1873047

Soft x-ray resonant magneto-optical Kerr effect as a depth-sensitive probe of magnetic heterogeneity: Its application to resolve helical spin structures using linear p polarization

J. Appl. Phys. 96, 7414 (2004); 10.1063/1.1806535

Proximity effects at epitaxial Co/FeMn thin film systems (invited)

J. Appl. Phys. 93, 6504 (2003); 10.1063/1.1555319

Contrasting hysteresis behavior between the magneto-optical Kerr rotation and ellipticity in NiFe/FeMn/Co trilayer films

J. Appl. Phys. 91, 3099 (2002); 10.1063/1.1435828

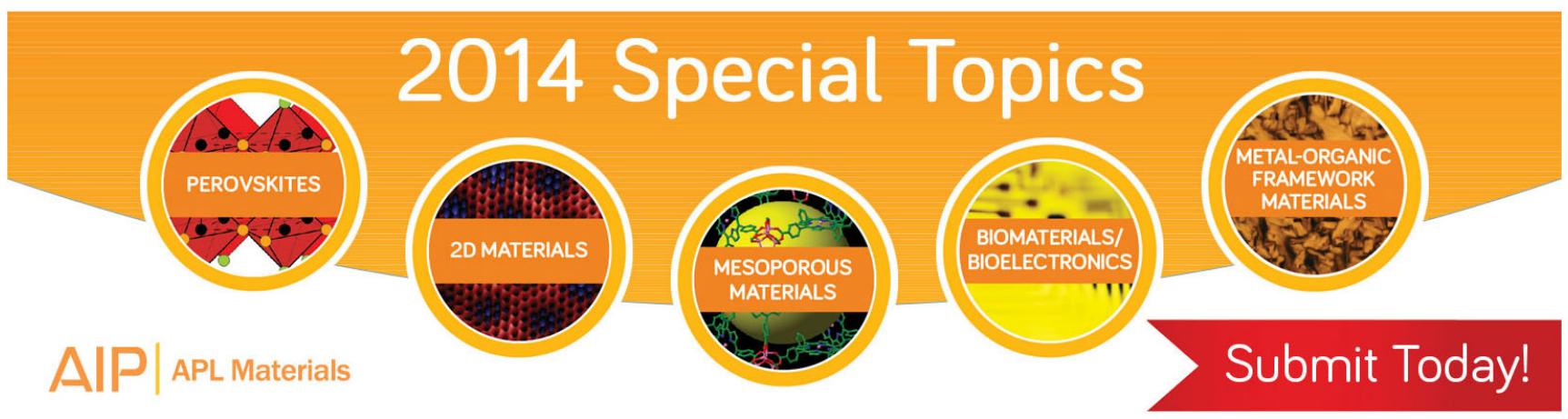




\title{
Atomic-scale depth selectivity of soft x-ray resonant Kerr effect
}

\author{
Ki-Suk Lee and Sang-Koog Kim ${ }^{\text {a) }}$ \\ Nanospintronics Laboratory, School of Materials Science and Engineering, and Research Institute of \\ Advanced Materials, College of Engineering, Seoul National University, Seoul 151-744, Korea \\ J. B. Kortright \\ Materials Sciences Division, Lawrence Berkeley National Laboratory, University of California, \\ One Cyclotron Road, Berkeley, California 94720
}

(Received 5 May 2003; accepted 28 August 2003)

\begin{abstract}
By the use of resonant soft $\mathrm{x}$-ray Kerr rotation measurements with its varying incident angle and energy, we observed various shifts of the exchange bias field of a 3.5-nm-thick Co layer in oppositely exchange-biased $\mathrm{Ni}_{81} \mathrm{Fe}_{19} / \mathrm{Fe}_{50} \mathrm{Mn}_{50} / \mathrm{Co} / \mathrm{Pd}$ films. The results in conjunction with their model simulations clearly reveal that the measurements enable one to resolve varying magnetization with depth in the individual magnetic layers of such a multicomponent ultrathin layered structure on the atomic scales. Significant interference effects combined with penetration depth of resonant soft $\mathrm{x}$ rays, which are closely associated with their absorptive and refractive contributions, offer remarkably different depth sensitivities into the Kerr effects depending on grazing angle and resonance energy. (c) 2003 American Institute of Physics. [DOI: 10.1063/1.1622123]
\end{abstract}

Current miniature magnetic devices, such as spin valve sensors and nonvolatile memory cells applicable to ultrahigh-density information storages, ${ }^{1}$ consist of complex multicomponent ultrathin layers having nanometer-scale thickness or less. The magnetic properties or functions of those films are known to be determined crucially by structural and magnetic properties at many interfaces and are known to be varying with depth in the individual layers. ${ }^{2-5}$ In order to better understand their intriguing properties associated with interfaces, it is essential to examine the depth dependence of magnetic information. However, many measurement techniques average various magnetic characteristics in the film plane.

There have been several attempts to separately investigate the magnetic properties of individual layers in ultrathin layered structures. ${ }^{6-9}$ In these works, the different magnetic properties of the individual layers have been resolved by visible or soft $\mathrm{x}$-ray magneto-optical Kerr effects. In earlier work, ${ }^{3}$ we also attempted to resolve depth-varying magnetic properties of a Co layer by the use of standing waves generated from a well-ordered multilayer on top of which the Co layer is deposited. Here we report that soft x-ray Kerr rotation, $\theta_{K}$, versus incident grazing angle, $\phi$, and energy, $h \nu$, measurements can provide an extremely large depth selectivity on the atomic scales even in a ultrathin single layer, simply by choosing appropriate $\phi$ and $h \nu$ around the resonant regions. For instance, both the experimental and simulation results of $\phi$ vs $\theta_{K}$ measurements are presented for depthvarying magnetization reversals in a 3.5-nm-thick Co layer of $\mathrm{NiFe} / \mathrm{FeMn} / \mathrm{Co} / \mathrm{Pd}$ films.

The sample of a structure of $\mathrm{SiO}_{2}(150 \mathrm{~nm}) / \mathrm{Ta}$ $(5 \mathrm{~nm}) / \mathrm{Ni}_{81} \mathrm{Fe}_{19}(8 \mathrm{~nm}) / \mathrm{Fe}_{50} \mathrm{Mn}_{50}(20 \mathrm{~nm}) / \mathrm{Co}(3.5 \mathrm{~nm})$ has oppositely oriented exchange bias between two ferromagnetic (F) NiFe and Co layers with an antiferromagnetic (AF) FeMn layer, as seen in visible Kerr loop shown in the inset of

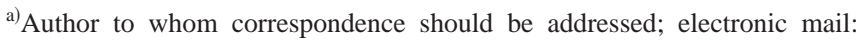
sangkoog@snu.ac.kr
}

Fig. 1(a). The oppositely oriented exchange bias was locked in by cooling through a Blocking temperature with their antiparallel remanent magnetizations oppositely saturated above the Blocking temperature in a high vacuum chamber with a base pressure of $2 \times 10^{-8}$ Torr. Details of the sample growth, representative characteristics, and exchange-bias setting will be reported elsewhere. ${ }^{10}$ We notice here that a Pd capping layer was deposited to prevent oxidation after the formation of the opposite exchange bias in $\mathrm{NiFe} / \mathrm{FeMn} / \mathrm{Co}$ films. After the Pd deposition, the Co magnetization reversal becomes nonuniform, rather shows varying exchange bias field, $H_{\mathrm{eb}}$, as seen in a vibrating sample magnetometer (VSM) loop [see Fig. 1(a)]. While, NiFe exchange bias remains unchanged after the Pd deposition since the NiFe layer is far away in distance from the Pd capping layer.

The nonuniform Co reversal is likely to be modified by Pd proximity effect on the Co surface. To clarify the true origin of the observed nonuniform reversals, we have employed a technique of resonant soft x-ray $\phi$ vs $\theta_{K}$ measurements that enable to resolve magnetization reversals of different elements and their depth varying reversals in complex multicomponent layered structures. The measurements were carried out on the beamline 8.0 at the Advanced Light Source at Lawrence Berkeley National Laboratory. The details have been described elsewhere. ${ }^{11}$

Figure 1(b) shows Co-resolved magnetization loops measured via soft x-ray $\theta_{K}$ at different $\phi$ as noted for somewhat different $h \nu$ around the Co $L_{3}$ edge. Various $H_{\mathrm{eb}}$, coercivity, $H_{c}$, and slopes of the loops are clearly seen depending on $\phi$. Those different values that are characteristic of each $\phi$ loop, are associated with different parts of the entire Co reversal shown in the VSM loop. Among those, $H_{\mathrm{eb}}$ for $\phi=7^{\circ}$ is almost zero, apparently indicating nonexchange biasing. For other $\phi$ loops, Co exchange biasing is present, and in particular, $H_{\mathrm{eb}}$ for $\phi=10^{\circ}$ is exactly the same as that of the Co reversal observed from that sample without Pd deposition. It is quite interesting how $\phi$ dependent $\theta_{K}$ loop measurements give almost narrow single switching loops with different $H_{\mathrm{eb}}, H_{c}$, and slopes that are resolved from the 


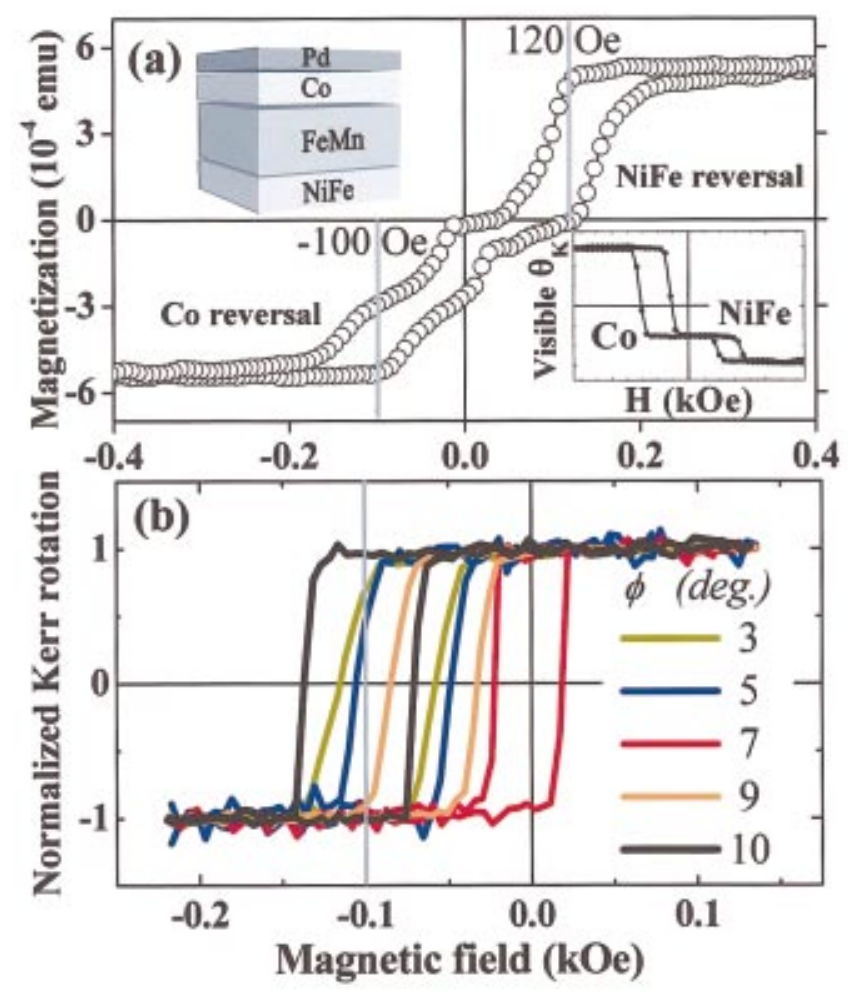

FIG. 1. (Color) (a) Magnetization reversals of NiFe and Co layers which are oppositely exchange biased to a FeMn layer. The lower inset shows magnetization reversals of both $\mathrm{NiFe}$ and Co layers measured with visible Kerr effect in a high vacuum chamber prior to deposition of a Pd capping layer. The two vertical gray lines indicate $H_{\mathrm{eb}}$ values for the Co $(-100 \mathrm{Oe})$ and $\mathrm{NiFe}(120 \mathrm{Oe})$ reversals. The visible Kerr loop has opposite sense in the magnitude and different sensitivity to layers. (b) Co resolved magnetization reversals measured via soft x-ray $\theta_{K}$ at different $\phi$ as noted and somewhat different $h \nu$ near the Co $L_{3}$ edge. The sign and magnitude of $\theta_{K}$ are normalized with the saturation value at a positive saturation field. The vertical gray line is placed at -100 Oe which is the same as $H_{\mathrm{eb}}$ of a part of the entire Co reversal shown in the VSM loop.

mixed, multiple Co switching shown in the VSM loop. Such measurements should inform on depth-varying heterogeneity in the magnetization reversals of a ultrathin Co layer with sufficient depth resolutions. ${ }^{12}$

In order to verify it, we calculate $\theta_{K}$ versus both $\phi$ and $h \nu$ from some model spin structures illustrated in Fig. 2(a). In this model, we divide the 3.5-nm-thick Co layer into three different regions of the same thickness and use a spin flip model where the magnetization directions of three different regions are independently flipped. For calculations of $\theta_{K}$ from the model structures around the Co $L_{3}$ and $L_{2}$ edges, $+/$ - helicity dependent complex refractive indices, $n_{+/-}(h \nu)$, should be used for Co, which are obtained from right and left circularly components of Co absorption spectra measured versus $h \nu$ and their Kramers-Kronig transformations. ${ }^{13}$ For $n(h \nu)$ of other layers such as NiFe, FeMn, Pd, etc., we use tabulated Henke data ${ }^{14}$ since the Co resonant region of interest in this calculation corresponds to their nonresonant $h \nu$ regions as for those elements. We also use the real part of $-r_{p s} / r_{s s}$ for $\theta_{K}$, where $r_{s s(p s)}$ is the reflection coefficient from $s(s)$ to $s(p)$ polarization.

From calculated $\theta_{K}$ against both $\phi$ and $h \nu$ from each spin configuration noted as (I), (II), (III), and (IV) in Fig. 2(a), their differences in Fig. 2(b) are remarkably contrasting

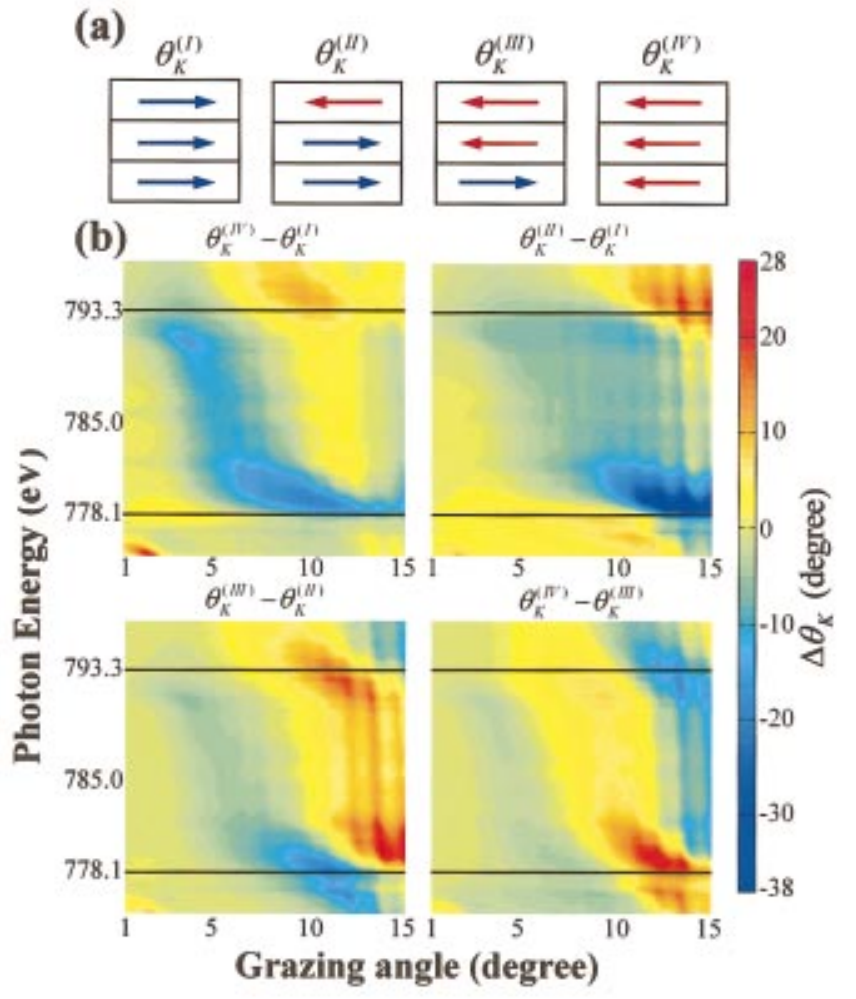

FIG. 2. (Color) (a) A spin flip model where the directions of spins placed at three different depths reverse incoherently in depth as schematically illustrated. (b) Calculated contrasts in $\theta_{K}$ for independent spin flips, plotted as contour against $\phi$ and $h \nu . \theta_{K}^{(\mathrm{IV})}-\theta_{K}^{(\mathrm{I})}$ represents the contrast in $\theta_{K}$ for a spin flip from one direction to the other opposite one coherently through the entire Co thickness, whereas $\theta_{K}^{(\mathrm{III})}-\theta_{K}^{(\mathrm{I})}, \theta_{K}^{(\mathrm{III})}-\theta_{K}^{(\mathrm{II})}$, and $\theta_{K}^{(\mathrm{IV})}-\theta_{K}^{(\mathrm{III})}$ correspond to the contrasts in $\theta_{K}$ for each of the individual spin flips at different top, middle, and bottom depths, respectively. (a) Coherent rotation
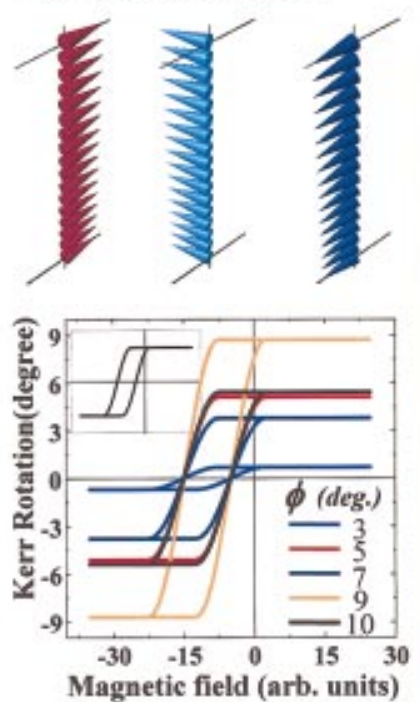

(b) Incoherent rotation
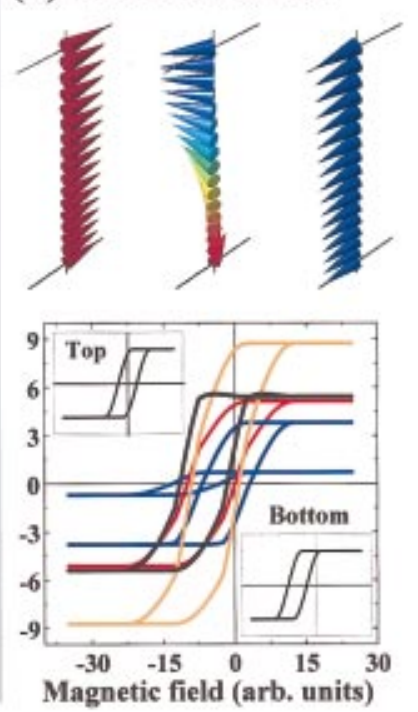

FIG. 3. (Color) Model spin structures within a Co layer for coherent and incoherent magnetization reversals and calculated $\theta_{K}$ loops based on the models. In (a), the inset shows the model hysteresis loop of a coherent reversal with a certain $H_{\mathrm{eb}}$ of the same value for all the individual spins. In (b), the upper and lower insets indicate the model hysteresis loops, respectively of top and bottom layer spins, which are assumed to have different $H_{\mathrm{eb}}$ as shown in the insets. 
at certain $\phi$ and $h \nu$ around the resonant regions for the independent spin flips, indicating that $\theta_{K}$ has a significant depth sensitivity. Also, the noticeable changes in the magnitude and sign of those contrasts on the $\phi-h \nu$ plane exhibit a large variation of the depth sensitivities of $\theta_{K}$ depending on $\phi$ and $h \nu$. This origin is ascribed to strong interference effects in conjunction with penetration depth that are associated closely with absorptive and refractive nature of soft $\mathrm{x}$ rays in response to such a complex multilayer film. ${ }^{13}$

It was revealed earlier that Co-specific $\theta_{K}$ is strongly sensitive to depth at which magnetization reversal occurs. This sensitivity is also distinctly different with varying $\phi$ and $h \nu$ near the Co absorption edges. With these regards, to verify that varying $H_{\mathrm{eb}}$ observed by soft x-ray $\phi$ vs $\theta_{K}$ is caused by depth varying exchange bias, we simulate $\theta_{K}$ loops for several different $\phi$ at certain $h \nu$ from two distinct models of coherent and incoherent rotations in depth within a single Co layer, as shown in Fig. 3. All the calculated $\theta_{K}$ loops at different $\phi$ and $h \nu$ from the coherent model represented by a hysteresis loop shown in the inset, exhibit the same $H_{\mathrm{eb}}$ and $H_{c}$ as expected, but different magnitudes and signs depending on $\phi$ and $h \nu$ because of their related interference effects. While, an incoherent rotation in depth shows a remarkable change in $H_{\mathrm{eb}}$ depending on $\phi$ or $h \nu$ because of the different depth sensitivities as a function of $\phi$ and $h \nu$, as evidently shown in Fig. 2. These confirm that for a uniform magnetization reversal, $\phi$ vs $\theta_{K}$ measurements do not show any change in $H_{\mathrm{eb}}$, whereas nonuniform magnetization reversals evidently yield changes in $H_{\mathrm{eb}}$ depending on $\phi .{ }^{15}$

The simulation results, hence, provide a clear evidence that the experimental result of depth-resolved exchange bias holds true and can be obtained from $\phi$ vs $\theta_{K}$ measurements. Here we notice that we neither intend to simulate the exact shapes of experimentally observed loops and nor intend to determine the exact spin structure in the Co layer during the reversal. The observed nonuniform exchange bias itself is quite interesting because the spin structure of a $F$ layer coupled to an AF layer is typically assumed to be uniform inside the $F$ layer in most previous models of exchange bias. Also, the nonuniform exchange bias inside the Co layer should give rise to incoherent rotation in depth during the reversal. In this work, even though we cannot determine the exact spin structure of Co, our experimental observation of depth-resolved, Co-specific loops supports that at least a part of spring-like (twist) spin structure might be present within the Co layer during the reversal. Probably, the incoherent rotation in such an ultrathin Co layer may be possible for the case of much reduced Co exchange coupling compared to that of the bulk. Not only Pd proximity but also many in situ measurements during the Co growth seem to cause such reduction in Co exchange coupling in our sample.

Finally, in order to examine to what extent depth resolution can be obtained in a single magnetic layer, we also calculate $\theta_{K}$ with varying $\phi$ from a simple $\mathrm{Co} / \mathrm{Pd}$ bilayer structure. Figure 4 shows the calculated contrasts in $\theta_{K}$ vs $\phi$ for Co spin flips independently at top, middle, and bottom layers of one monolayer thickness. The contrast at $\phi=6.0^{\circ}$ is almost zero for the spin flip of the bottom layer, while largest for the top spin flip. Thus, at that angle any spin reversal at

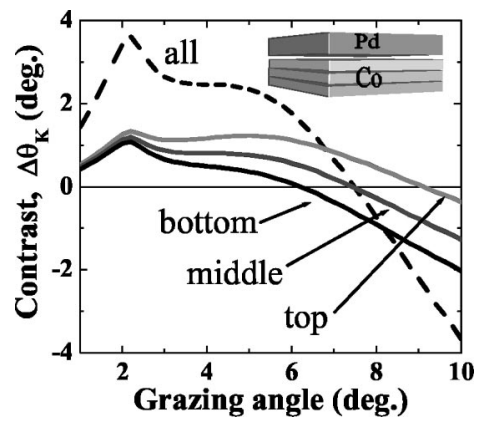

FIG. 4. Calculated contrasts in $\theta_{K}$ as a function of $\phi$ at $h \nu=778.5 \mathrm{eV}$ for spin flips independently at top, middle, and bottom depths within the 0.6nm-thick Co layer of a Co $(0.6 \mathrm{~nm}) / \mathrm{Pd}(1.5 \mathrm{~nm})$ bilayer structure.

the bottom layer cannot be measured, but the top spin reversal can be resolved sensitively via $\theta_{K}$ since its largest sensitivity. In contrast, the depth selectivity at $\phi=9.2^{\circ}$ is reversed for the top and bottom spin reversals. By choosing not only appropriate $\phi$ but also $h \nu$ in the $\theta_{K}$ measurements, thus we can resolve magnetization reversals at different depths even in a few monolayer thick layers.

This work was supported by the KOSEF through q-Psi and Korea Research Foundation Grant No. KRF-2002-003D00137. Work at the ALS was supported by the Director of the U.S. Department of Energy under Contract No. DEAC03-76SF00098.

${ }^{1}$ G. A. Prinz, Science 282, 1660 (1998).

${ }^{2}$ H. Ohldag, T. J. Regan, J. Stohr, A. Scholl, F. Nolting, J. Luning, C. Stamm, S. Anders, and R. L. White, Phys. Rev. Lett. 87, 247201 (2001).

${ }^{3}$ S.-K. Kim and J. B. Kortright, Phys. Rev. Lett. 86, 1347 (2001).

${ }^{4}$ S. H. Yang, B. S. Mun, N. Mannella, S.-K. Kim, J. B. Kortright, J. Underwood, F. Salmassi, E. Arenholz, A. Young, Z. Hussain, M. C. Van Hove, and C. S. Fadley, J. Phys.: Condens. Matter 14, L407 (2002).

${ }^{5}$ J. L. Prieto, M. G. Blamire, and J. E. Evetts, Phys. Rev. Lett. 90, 027201 (2003).

${ }^{6}$ O. Hellwig, J. B. Kortright, K. Takano, and E. E. Fullerton, Phys. Rev. B 62, 11694 (2000).

${ }^{7}$ J. Hamrle, J. Ferre, M. Nyvlt, and S. Visnovsky, Phys. Rev. B 66, 224423 (2002).

${ }^{8}$ G. Penissard, P. Meyer, J. Ferre, and D. Renard, J. Magn. Magn. Mater. 146, 55 (1995).

${ }^{9}$ J. Ferre, P. Meyer, M. Nyvlt, S. Visnovsky, and D. Renard, J. Magn. Magn. Mater. 165, 92 (1997).

${ }^{10}$ K.-S. Lee, S.-K. Kim, J. B. Kortright, K. Y. Kim, Y.-W. Kim, and S.-C. Shin (unpublished).

${ }^{11}$ J. B. Kortright, M. Rice, S.-K. Kim, C. C. Walton, and T. Warwick, J. Magn. Magn. Mater. 191, 79 (1999).

${ }^{12}$ We believe that the size of soft $\mathrm{x}$-ray beam is much larger than that of many magnetic domains of somewhat different $H_{\mathrm{eb}}$ so that the measured Kerr signal is average over slightly different $H_{\mathrm{eb}}$ of the individual domains at each $\phi$. If experimentally observed nonuniform exchange bias were caused by a possible lateral heterogeneity, every $\phi$ loop would show nonuniform reverslas as well, as observed by a VSM.

${ }^{13}$ J. B. Kortright and S.-K. Kim, Phys. Rev. B 62, 12216 (2000).

${ }^{14}$ B. L. Henke, E. M. Gullikson, and J. C. Davis, At. Data Nucl. Data Tables 54, 181 (1993); http://www-cxro.lbl.gov

${ }^{15}$ In this model, while $H_{\mathrm{eb}}$ varies with depth, $H_{c}$ and slopes for different depths are assumed to be the same. Therefore, $H_{c}$ does not vary with $\phi$, but the loop slopes somewhat change with depth since different $H_{\text {eb }}$ with depth assumed in the model contribute to each loop at a given $\phi$. In experimental results, the variations in $H_{c}$ and slope as well as $H_{\mathrm{eb}}$ with $\phi$ are possible due to likely depth varying $H_{c}$ in the Co layer of the sample used in this work. 